\title{
Measuring the Mappability Spectrum of Reference Genome Assemblies
}

\author{
Zachary D. Stephens \\ University of Illinois at Urbana-Champaign \\ Champaign, IL, USA \\ zstephe2@illinois.edu
}

\author{
Ravishankar K. Iyer \\ University of Illinois at Urbana-Champaign \\ Champaign, IL, USA \\ rkiyer@illinois.edu
}

\begin{abstract}
The ability to infer actionable information from genomic variation data in a resequencing experiment relies on accurately aligning the sequences to a reference genome. However, this accuracy is inherently limited by the quality of the reference assembly and the repetitive content of the subject's genome. As long read sequencing technologies become more widespread, it is crucial to investigate the expected improvements in alignment accuracy and variant analysis over existing short read methods. The ability to quantify the read length and error rate necessary to uniquely map regions of interest in a sequence allows users to make informed decisions regarding experiment design and provides useful metrics for comparing the magnitude of repetition across different reference assemblies.

To this end we have developed NEAT-Repeat, a toolkit for exhaustively identifying the minimum read length required to uniquely map each position of a reference sequence given a specified error rate. Using these tools we computed the "mappability spectrum" for ten reference sequences, including human and a range of plants and animals, quantifying the theoretical improvements in alignment accuracy that would result from sequencing with longer reads or reads with less base-calling errors. Our inclusion of read length and error rate builds upon existing methods for mappability tracks based on uniqueness or aligner-specific mapping scores, and thus enables more comprehensive analysis.

We apply our mappability results to whole-genome variant call data, and demonstrate that variants called with low mapping and genotype quality scores are disproportionately found in reference regions that require long reads to be uniquely covered. We propose that our mappability metrics provide a valuable supplement to established variant filtering and annotation pipelines by supplying users with an additional metric related to read mapping quality.

NEAT-Repeat can process large and repetitive genomes, such as those of corn and soybean, in a tractable amount of time by leveraging efficient methods for edit distance computation as well as running multiple jobs in parallel. NEAT-Repeat is written in Python 2.7 and $\mathrm{C}++$, and is available at https://github.com/zstephens/neat-repeat.
\end{abstract}

Permission to make digital or hard copies of all or part of this work for personal or classroom use is granted without fee provided that copies are not made or distributed for profit or commercial advantage and that copies bear this notice and the full citation on the first page. Copyrights for components of this work owned by others than the author(s) must be honored. Abstracting with credit is permitted. To copy otherwise, or republish, to post on servers or to redistribute to lists, requires prior specific permission and/or a fee. Request permissions from permissions@acm.org.

ACM-BCB'18, August 29-September 1, 2018, Washington, DC, USA

(C) 2018 Copyright held by the owner/author(s). Publication rights licensed to ACM.

ACM ISBN 978-1-4503-5794-4/18/08 ..\$15.00

https://doi.org/10.1145/3233547.3233582

\section{CCS CONCEPTS}

- Applied computing $\rightarrow$ Computational genomics; Molecular sequence analysis;

\section{KEYWORDS}

Sequence analysis, repetitive dna, mappability

ACM Reference Format:

Zachary D. Stephens and Ravishankar K. Iyer. 2018. Measuring the Mappability Spectrum of Reference Genome Assemblies. In ACM-BCB'18: 9th ACM International Conference on Bioinformatics, Computational Biology and Health Informatics, August 29-September 1, 2018, Washington, DC, USA. ACM, New York, NY, USA, 6 pages. https://doi.org/10.1145/3233547.3233582

\section{INTRODUCTION}

It is well known that the genomes of most organisms are highly structured and nonrandom. Genome sequences contain a substantial number of repeated elements, and large repetition limits the ability of a resequencing experiment to determine from which region a fragment of DNA may have originated.

Repetitive DNA elements can range from very short, such as those found in tandem repeats or satellite DNA, up to tens of thousands of nucleotides, such as large transposable elements. Determining the functional significance of repetitive DNA is an active field of research, with applications spanning medicine $[9,11]$, phylogeny [4], evolution studies [2, 15, 31], forensics [3], environmental science [7], and general understanding of genome function [30, 33]. Investigation of these repetitive elements with high-throughput sequencing data is challenging due to the inherent limitations of short read data. There is significant enthusiasm for emerging long read technologies, as well as paired-end (PE) sequencing methods with large fragment lengths, which will generate data capable of uniquely mapping more of a genome than currently prevalent methods (e.g. reads of length 100 to 150) are able to. In this work, we present a method for further quantifying the relationship between read length and reference mappability. With this method, we exhaustively identify positions of a reference genome that are uniquely mappable as a function of read length and error rate. We performed this analysis on ten reference sequences, including human and a range of plants and animals.

There has been significant work in attempting to identify and quantify repetitive DNA in the genomic sequences. Many specialized tools exist for detecting certain classes of repeats, including tandem repeats $[1,12]$, satellite DNA $[8,27]$, or interspersed transposable elements such as Alus [8, 13, 29]. Other software, such as RepeatMasker [32] work by comparing an input sequence to a library of known repetitive elements specified a priori, but such 
methods are less helpful for analyzing highly divergent sequences or organisms whose genomes are not well documented.

Many existing tools for detecting arbitrary interspersed and imperfect repetition tend to use approximate string-matching methods. Vmatch [16] (formerly REPuter [17, 18]) is capable of exhaustively searching a DNA sequence for maximal repeats, accounting for imperfect and reverse complement matching. Another class of tools utilize the statistics produced by alignment software to compute reference mappability [14, 19]. The GEM suite of programs [6] accomplishes a similar goal of computing per-base mappability scores using an approximate mapping strategy.

We have developed a tool, NEAT-Repeat, which combines exhaustive search and seed-and-extend methods to uncover all large-scale repetition in an input sequence. Using this tool, we identify the minimum required read length to uniquely map each position in a reference genome. From this information, we can compute a "mappability spectrum" describing the proportion of unmappable coordinates in a reference. Our tool builds on existing kmer-based methods for sequence analysis [22, 24] with the added flexibility of allowing kmer size and error rate to be abitrarily large. The mappability tracks generated by our tools can be visualized in genome browsers much like existing tracks, but are more comprehensive in that they explicitly identify the read length required to uniquely cover each unmappable position. Currently, our analysis is focused on single-end (SE) reads of arbitrary length, but the methodology is extensible to paired-end reads of fixed read and fragment lengths.

The primary contribution of this work is the quantification of uniquely mappable reference coordinates for any read length and any maximum edit distance. We apply NEAT-Repeat to a multitude of reference assemblies, providing a way to compare their proportion of mappable positions. Additionally, we process two human reference assemblies (GRCh37 and GRCh38) to estimate the utility of long reads with high error rates.

\section{BACKGROUND}

Given a string $S$, we call a substring $S[i: j]$ (spanning elements $\left.S_{i} \ldots S_{j-1}\right)$ repetitive if and only if $\exists x, y \mid[i, j] \cap[x, y]=\emptyset$, and $S[i: j]=S[x: y]$. A repeat $S[i: j]$ is maximal if and only if neither $S[i-1: j]$ nor $S[i: j+1]$ is a repeat. To make it applicable to mappability studies, we extend the basic definition as follows:

- Length constraint: We are interested in the repetitive content of DNA sequences as a function of repeat size, so we assert that our detected repeats must be at least a minimum length $L$.

- Imperfect repeats: To account for the presence of sequencing errors, genetic variation, and the mismatches tolerated by alignment software, we relax the definition to allow differences. We consider two strings repetitive if they differ by an edit distance no more than $E$. We use $D\left(s_{1}, s_{2}\right)$ to denote the edit distance between two strings $s_{1}$ and $s_{2}$.

- Reverse complementation: To account for the dual-strandednes of DNA, we consider a substring repetitive if its reverse complement is found elsewhere. We use $\bar{s}$ to denote the reverse complement of a string $s$.

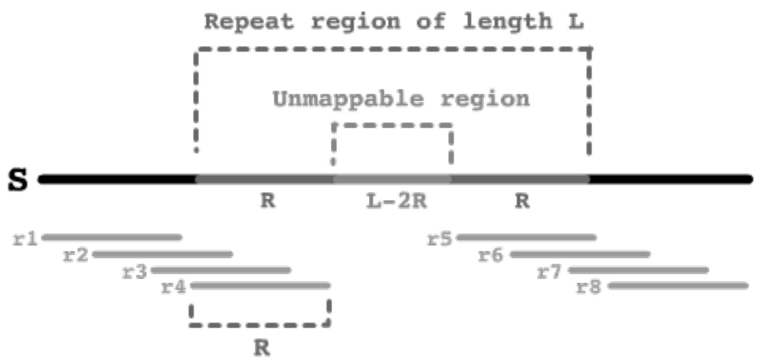

Figure 1: An example repetitive region in a reference string $S$ with aligned reads of length $R$. The unmappable region is the center $L-2 R$ nucleotides that cannot be covered by a uniquely mapped read.

Therefore, per our definition, $S[i: j]$ is determined to be nonunique if and only if:

$$
\begin{aligned}
& \exists x, y \mid[i, j] \cap[x, y]=\emptyset \wedge \\
& \quad(D(S[i: j], S[x: y]) \leq E \vee D(S[i: j], \overline{S[x: y]})) \leq E)
\end{aligned}
$$

A substring $S[i: j]$ is unmappable by SE reads of length $\mathrm{R}$ if none of its positions can be covered by a uniquely mappable read (Fig. 1). That is, for a maximal repeat $S[i: j]$, nucleotide $S_{k}(i<k<j)$ is unmappable by reads of length $\mathrm{R}$ if $\nexists x \mid((x<i) \wedge(x+R \geq k))$ $\vee((x<k) \wedge(x+R \geq j))$. It follows that nucleotides unmappable by length-R reads must be contained within a maximal repeat of length $>2 R$. That is, the nucleotides within the center of a repeat of length $>2 R$ are unmappable due to the other occurrences of the repeat.

\section{METHODS}

NEAT-Repeat utilizes an exhaustive seed-and-extend approach to identify nearly all self-similarity in a genomic sequence (i.e. all pairs of repeated sequences that contain an exact match of some size $K$, and are within an edit distance $E$ of each other). At each step of analysis we leverage existing methods for efficient kmer finding and seed extension, and the computationally demanding portions of the analysis are heavily parallelized. These optimizations allow us to analyze very large or very repetitive genomes in a tractable amount of time.

\section{Seed Finding}

To identify the seed kmers of length $K$ that occur within a reference at least two times we use the popular kmer-counting tool Jellyfish [25]. We selected a value of $K=40$, under the assumption that nearly all the large repeats in which we are interested will contain at least one exact match of length 40 . Because any two occurrences of a repeat of size $L$ (allowing edit distance $E$ ) contain an exact match of at least size $\left\lfloor\frac{L}{(E+1)}\right\rfloor[17]$, with a seed size of $K=40$ we are guaranteed to find every repeat of size $\geq 40(E+1)$.

By choosing $K=40$ it is possible that we may miss certain smaller repeats if the differences in each occurrence are distributed such that no exact match of length 40 exists. We expect such cases to be rare. In a preliminary analysis of Arabidopsis thaliana we swept 
over $K$ values from 30 to 50 , and found there to be no significant difference in the proportion of the genome identified as unmappable at $E=0$. If we allowed a $1 \%$ error rate between matches $(E=$ $0.01 \times L$ ), we identified approximately $0.2 \%$ more of the genome as unmappable with $K=30$ as compared to $K=50$.

\section{Seed Extension}

For each non-unique kmer, we enumerate all $N$ reference positions where the kmer or its reverse complement are found. These positions are then aligned pairwise until the specified edit distance has been reached, yielding $N(N-1) / 2$ coordinate boundaries that can be compiled into a mappability track. If we wish to define repeats such that the permitted number of edits is proportional to the length of the region we are extending (e.g. to reflect an "error rate" such as the number of base-calling errors in a read of sequenced DNA) then we reevaluate the maximum edit distance after each successful boundary extension, increasing the threshold as the boundaries grow. To reduce the number of redundant seed extensions, all pairwise alignments for all non-unique kmers are first performed with edit distance 0 (i.e. stopping at the first mismatch). Duplicates are then removed, corresponding to pairs of different kmers that would be extended to the same coordinate boundaries. The pruned boundaries are then fully extended until the specified edit distance is reached.

Our choice to pursue computationally intensive pairwise alignments instead of performing seed extension on consensus sequences, as in RepeatScout [28], is because our primary interest with NEATRepeat is not in cataloging frequently repeated elements (where the consensus-derived coordinate boundaries are more informative than pairwise maximally-extended boundaries), but rather to interrogate all regions of a genomic sequence and estimate the minimum read length and error rate required to uniquely map them.

\section{Mappability Tracks}

A mappability track is a useful way to specify regions within a reference sequence that are challenging for genome assembly and read alignment due to repetition. In existing work, mappability tracks have been generated by using BWA to map reference kmers back to the entire reference sequence and using the alignment statistics (e.g., coverage and mapping quality) to compute a relative mapping score [19]. This approach has the advantage of empirically incorporating sequencing errors and aligner heuristics, so we expect the unmappable positions identified this way to be a superset of the theoretically unmappable regions found by NEAT-Repeat.

Another approach taken by Derrien et al. [6] uses the "k-uniqueness" of each reference position as a proxy to mappability. This metric describes the number of times a kmer at a given position is found elsewhere in the reference (within a certain edit distance). This is a useful estimation of mappability that can be quickly computed, but is different from a truly unmappable position that cannot be spanned by any unique kmers. The authors note that the relationship between mappability and uniqueness becomes increasingly complex when imperfect repetition is included. That is, when two reference kmers $s_{1}, s_{2}$ are within an edit distance $E$ of each other, a read within distance $E$ of $s_{1}$ may be anywhere between $[0,2 E]$ away from $s_{2}$. Thus, it is important to consider that regions identified as

\begin{tabular}{|r|ll|}
\hline Species: & Build: & Length (Mbp): \\
\hline Arabidopsis thaliana & 10 & 120 \\
Caenorhabditis elegans & WBcel235 & 100 \\
Gallus gallus & galGal5 & 1,021 \\
Glycine max & v1.0 & 950 \\
Homo sapiens & GRCh38 & 3,088 \\
Mus musculus & GRCm38.p5 & 2,725 \\
Oryza sativa & IRGSP-1.0 & 373 \\
Rattus norvegicus & rn6 & 2,782 \\
Saccharomyces cerevisiae & R64-1-1 & 12 \\
Zea mays & AGPv4 & 2,107 \\
\hline
\end{tabular}

Table 1: Overview of reference assemblies.

unmappable at high edit distances via pairwise comparisons may be overestimated.

NEAT-Repeat mappability tracks are populated with integer data specifying the minimum SE read length required to uniquely map each reference position. The values are computed by processing the extended seeds and noting the distance of each position from the nearest boundary. For example, the $i$ th position of a non-unique 1000 -mer is unmappable by SE reads of length $\leq \min \{i, 1000-i\}$. Tracks can be generated with fixed edit distances between matches (yielding tracks similar to "k-uniqueness"), or with constant error rates such that the permitted edit distance between matches scales with their length.

Mappability tracks for PE reads of length $R$ with fragment length $F$ (insert size $I=F-2 R$ ) can be estimated from SE tracks by sliding windows along non-unique positions of the track (repeat size $\geq R$ ), with indices corresponding to the reads $r_{1}=\mathrm{S}[\mathrm{i}: \mathrm{i}+\mathrm{R}]$, and $r_{2}=$ $\mathrm{S}[\mathrm{i}+\mathrm{R}+\mathrm{I}: \mathrm{i}+\mathrm{F}]$. The strings $r_{1}+\overline{r_{2}}$ and $\overline{r_{1}}+r_{2}$ are hashed, and we note the positions of all nucleotides belonging to any such strings that appear more than once. Finally, we prune out all the mappable nucleotides that can be covered by a PE read that spans the nonunique region but is anchored in a unique region. The limitations of this approach are that we cannot consider imperfect repetition, and fragment lengths are restricted to fixed values instead of distributions, which is unrealistic for real applications. It is for these reasons that we choose to estimate the mappability of $\mathrm{PE}$ reads from SE mappability tracks, using values corresponding to the average fragment length.

\section{RESULTS AND DISCUSSION}

Using NEAT-Repeat, we processed the reference assemblies of ten organisms from a range of plants and animals (Table 1). We included several plant genomes that contain a large number of repeats (G. max, Z. mays), as well as several model organisms with small genomes that are known to have a smaller proportion of repeats (S. cerevisiae, A. thaliana, C. elegans). For small references, NEATRepeat completes in only a few minutes. For the larger references, the computation time heavily depends on the error rate specified. For example, processing the human reference with error rate $0.5 \%$ took about $100 \mathrm{CPU}$ hours (on an Intel Xeon E5-2697 @ 2.7GHz). Finding imperfect repetition with a large number of mismatches is more demanding. For example, processing the human reference 

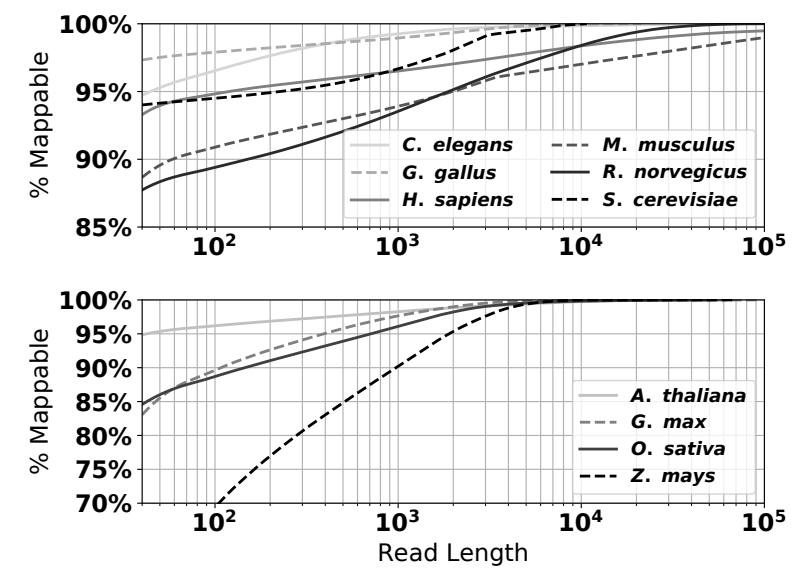

Figure 2: Mappability spectrum for each species with a fixed error rate of $1 \%$. Animalia/Fungi on top, Plantae on bottom. The y-axis of the bottom plot is extended to $70 \%$ to show the larger number of unmappable positions in plant references at short read lengths.

with a error rate of $10 \%$ required a total of $\sim 10,000$ CPU hours which we split into hundreds of parallel processes.

In addition to the standard chromosomal sequences, many references include a set of additional scaffolds and alternate assemblies. In this work we have excluded such sequences from the analysis.

It is important to note that the mappability values we present are derived solely from the repetitive content present in the reference, and thus are heavily influenced by the sequencing techniques used to derive and compile the assembly. Similarly, reference assemblies do not always fully characterize the true sequence of an organism, and may omit certain highly variable regions, or regions that are difficult to sequence or assemble. As an example, our mappability analysis of $C$. elegans suggests that $\geq 99.8 \%$ of the reference is uniquely mappable by reads of length 10,000 , but recent experiments [21] with reads of that length yielded an empirical genome coverage of $\sim 97 \%$, suggesting that the complexities of working with real sequencing data (including imperfect or heuristic alignment algorithms) reduce the practically attainable read alignment accuracy to below the estimates produced by our analysis. For that reason, we interpret our mappability results as an idealized upper bound of attainable read alignment accuracy for a particular reference assembly.

In Fig. 2 we show the "mappability spectrum" of each reference. We define mappability spectrum as the unmappable percentage of a reference sequence as a function of read length (and a specified error rate). This definition builds upon existing metrics for read uniqueness, such as k-uniqueness [6] or unique read percentage [5], in that we consider all read lengths (up to the size of the largest repeat) and higher error rates.

Mappability spectra are computed from our mappability tracks by counting the proportion of track values $\geq R$, for all read lengths $R$ present in the track. In general we notice a power law relationship between read length and unmappable positions, similar to previously observed power law relationships between reference kmer length and frequency [23]. The plant genomes tend to contain a

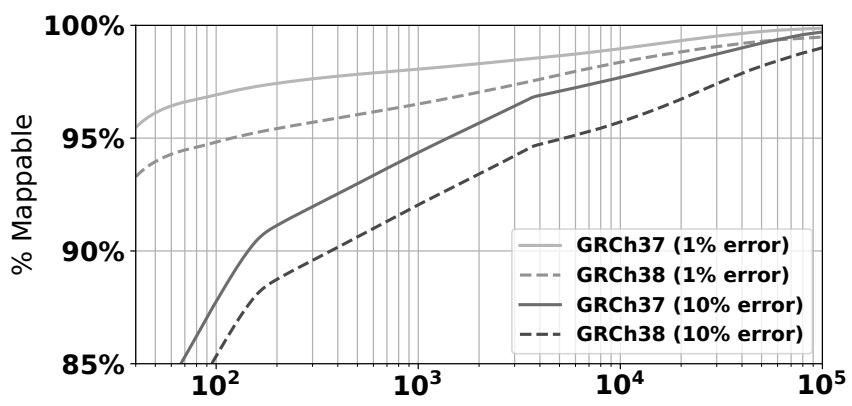

Figure 3: Mappability spectrum for human references GRCh37 and GRCh38, for fixed error rates $1 \%$ and $10 \%$.

very large number of small repeats, whereas the animal references tend to be less repetitive on average but contain significantly more very large repeats. For example, length-100 SE reads with $1 \%$ error rate can uniquely map only $\sim 90 \%$ of the G. max and $O$. sativa references, and a mere $70 \%$ of $Z$. mays, but can map $95 \%$ of $H$. sapiens. Longer reads of length 5,000 are able to map $99.9 \%$ of the plant genomes, but only $98 \%$ of the human genome.

Each reference exhibited a unique particular shape in their mappability spectrum. For example, we expected that the spectrum of $R$. norvegicus (rat) would be similar to that of M. musculus (mouse), given that the frequency and characteristics of known repetitive elements have been shown to be comparable [10], but we observe that $R$. norvegicus appears to have a larger number of small repeats, and fewer very large repeats. We speculate that the methods used to construct the rat assembly (e.g. the inclusion of PacBio data in addition to shotgun sequencing and bacterial artificial chromosomes) may have contributed to this observation, but a more detailed investigation is necessary.

\section{Mappability of GRCh37 vs. GRCh38}

By permitting a non-zero edit distance between repeats, we can produce a proxy for the maximum alignment accuracy that can be achieved with alignment software that allows mismatches and indels. In real read data, the number of errors scales with the length of the reads. To mimic this, NEAT-Repeat can create mappability tracks with proportional error rates in addition to fixed edit distances: extending all pairs of seeds until the number of mismatches between them exceeds a specified proportion of their length.

We processed human reference GRCh37, and the newer GRCh38, with error rates of $1 \%$ and $10 \%$ (Fig. 3). For both references we used primary chromosomal sequences only, omitting all alternative assemblies from our analysis. The $1 \%$ curve could represent the mapping power of reads with low error rates (e.g. Illumina short reads or error-corrected PacBio reads), and the $10 \%$ curve could represent the mapping power of reads with very high error rates. From this data we immediately observe that GRCh38 has approximately twice as many non-uniquely mappable coordinates at every read length for error rate $1 \%$, attributable largely to its increased representation of repetitive centromere regions (including modeled regions that estimate complex centromere structures, but may not be exactly representative of true centromere sequences [26]). At 


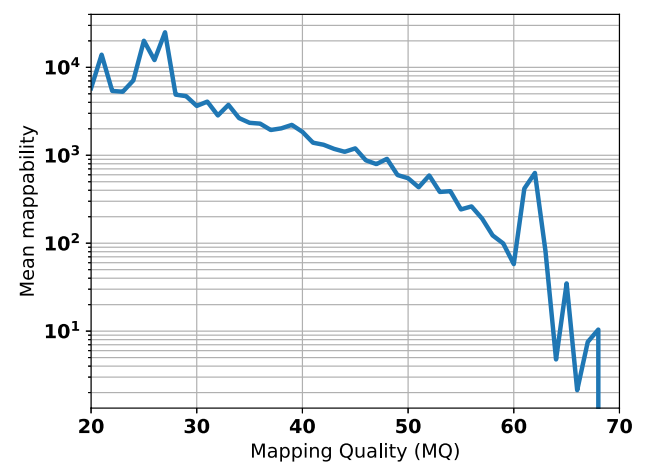

Figure 4: Mean mappability track value as a function of variant mapping quality score.

$10 \%$ error rate the difference between references is present, but proportionately less pronounced than at $1 \%$. This observation suggests that the non-unique sequences introduced in GRCh38 occur with high similarity (i.e. they do not require large edit distances to deem them a repeat).

From these mappability spectra we can estimate the trade-off of using long reads with high error rates as opposed to short reads with low error rates. For example, reads of length 20, 000 with $10 \%$ error can uniquely map $96.2 \%$ of GRCh 38 , which is comparable to using short reads with fragment length 500 which uniquely map $95.9 \%$ of the same reference.

\section{Mappability as a Predictor of Genotype and Mapping Quality}

To investigate the relationship between reference mappability and variant quality metrics, we intersected GRCh38 mappability tracks generated by NEAT-Repeat with a collection of 170 whole genome VCF files produced in-house by a GATK-based variant calling workflow for another study. PE libraries were prepared with TruSeq DNA v2 sample prep kits, following Illumina protocol. Length 101 paired-end sequencing was performed on Illumina HiSeq 2000s, and the reads were aligned to human reference GRCh38 using BWAMEM [20] (Again, excluding alternate assemblies). Duplicate reads were marked with Picard, and quality score recalibration was performed using GATK 3.3-0. Finally, variants were called using GATK HaplotypeCaller, resulting in 33,386,059 sites called across all 170 samples.

Fig. 4 shows the average mappability track values as a function of RMS Mapping Quality score (MQ) produced by HaplotypeCaller. We see that for variants with very high scores $(\mathrm{MQ} \geq 68)$, the average mappability is virtually zero, indicating that nearly every highscoring position was within genomic regions that were uniquely mappable with reads smaller than the seed size used to generate the tracks (40bp). As expected, we see that in general the MQ score of a variant is inversely related to the size of the repeat that it resides in, suggesting that reference repetition is a major contributor to mapping quality. We note that there are many sites that despite being located in positions that are theoretically unmappable by our

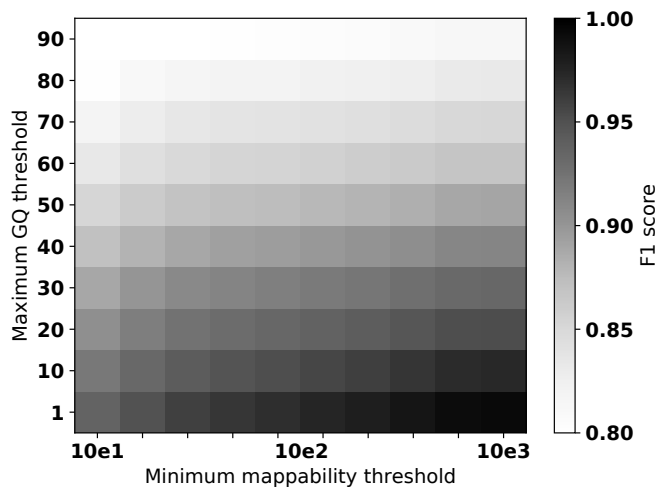

Figure 5: Accuracy for predicting that variant genotype quality (GQ) will be below some threshold given that mappability is above some value. We swept over GQ thresholds are from 1-90, and mappability thresholds from 100 to 10,000 .

reads (e.g. mappability $>1,000$ ) are still called with very high MQ scores. This could be due to a variety of factors, including heuristics used during alignment and variant calling, as well as the fact that the reference genome is not being perfectly representative of the sequenced individuals.

Variant Genotype Quality (GQ) is also potentially affected by reference mappability (Fig. 5). We observe that given the mappability of a site (the minimum read length required to uniquely map that position) is above some value, it is very likely that the GQ at that site is low. For example, from Fig. 5 we see that if the mappability of a site is above 100, then we can predict that the GQ will be below 10 with accuracy 0.92 (F1-Score). This relationship is expected, given that we observe MQ scores to be correlated with mappability, and that HaplotypeCaller computes genotype likelihoods using reads that pass internal mapping quality filters. However, because we do not observe reference mappability to be perfectly correlated with existing variant quality metrics produced by GATK HaplotypeCaller, we propose it is a valuable supplementary metric for use in variant filtering and annotation.

\section{CONCLUSION}

We have presented NEAT-Repeat, a methodology for exhaustive enumeration of repetitive and unmappable regions in DNA string data in order to generate mappability statistics and mappability tracks. From these data, we produce a "mappability spectrum" for a reference sequence, describing the relative amounts of imperfect genomic repetition as a function of repeat length and number of edits. Our mappability tracks can provide a proxy for the maximum attainable read alignment accuracy for a given read length and error rate. Unlike existing mappability tracks, our tracks specify the minimum read length necessary to uniquely map each position of a reference sequence.

As expected, we observe that an increase in read length can drastically increase the number of uniquely mappable positions, particularly for larger reference sequences. By computing the mappability spectrum at high edit distances or large fixed error rates, we 
estimate the mappability trade-offs between short reads and long reads with high error rates. We examine human reference sequences GRCh 37 and GRCh 38 at $1 \%$ and $10 \%$ error rates, and quantify the differences in proportion of uniquely mappable positions. We show that GRCh38 has about twice the amount of non-unique coordinates as compared to GRCh37, and that mapping reads of length 10,000 with $10 \%$ error rate have roughly the same power as reads of length 100 with $1 \%$ error.

Via intersecting NEAT-Repeat mappability tracks with variant call data we observe a relationship between low mapping and genotype quality scores and high mappability values, and suggest that mappability metrics are a useful supplement to established variant filtering and annotation methods.

NEAT-Repeat is written in $\mathrm{C}++$ and Python 2.7. Source code and mappability tracks (as quantized bed files) for each organism presented in this study are available at https:/github.com/zstephens/ neat-repeat.

\section{ACKNOWLEDGMENTS}

This material is based upon work partially supported by a CompGen Fellowship; an IBM Faculty Award; National Science Foundation (NSF) under grants CNS 13-37732, CNS 16-24790 and CNS 16-24615; and the Mayo Clinic Center for Individualized Medicine. Any opinions, findings, and conclusions or recommendations expressed in this material are those of the author(s) and do not necessarily reflect the views of the NSF. We thank Dr. Matthew Hudson at the University of Illinois Department of Crop Sciences for providing insightful commentary during this study.

\section{REFERENCES}

[1] Gary Benson. 1999. Tandem repeats finder: a program to analyze DNA sequences. Nucleic acids research 27, 2 (1999), 573.

[2] Roy J Britten and Eric H Davidson. 1971. Repetitive and non-repetitive DNA sequences and a speculation on the origins of evolutionary novelty. Quarterly Review of Biology (1971), 111-138.

[3] John M Butler et al. 2007. Short tandem repeat typing technologies used in human identity testing. Biotechniques 43, 4 (2007), $2-5$.

[4] Teresa Capriglione. 2000. Repetitive DNA as a tool to study the phylogeny of cold-blooded vertebrates. In Chromosomes Today. Springer, 183-194.

[5] Rayan Chikhi. 2012. Computational methods for de novo assembly of nextgeneration genome sequencing data. Ph.D. Dissertation. École normale supérieure de Cachan-ENS Cachan.

[6] Thomas Derrien, Jordi Estellé, Santiago Marco Sola, David G Knowles, Emanuele Raineri, Roderic Guigó, and Paolo Ribeca. 2012. Fast computation and applications of genome mappability. PloS one 7, 1 (2012), e30377-e30377.

[7] Priscilla E Dombek, LeeAnn K Johnson, Sara T Zimmerley, and Michael J Sadowsky. 2000. Use of repetitive DNA sequences and the PCR to differentiate Escherichia coli isolates from human and animal sources. Applied and Environmental Microbiology 66, 6 (2000), 2572-2577.

[8] Robert C Edgar and Eugene W Myers. 2005. PILER: identification and classification of genomic repeats. Bioinformatics 21, suppl 1 (2005), i152-i158.

[9] Jörg T Epplen. 1992. Diagnostic applications of repetitive DNA sequences. Clinica chimica acta 209, 3 (1992), S5-S13.
[10] Richard A Gibbs, George M Weinstock, Michael L Metzker, Donna M Muzny, Erica J Sodergren, Steven Scherer, Graham Scott, David Steffen, Kim C Worley, Paula E Burch, et al. 2004. Genome sequence of the Brown Norway rat yields insights into mammalian evolution. Nature 428, 6982 (2004), 493-521.

[11] Syed Haider, Lina Cordeddu, Emma Robinson, Mehregan Movassagh, Lee Siggens, Ana Vujic, Mun-Kit Choy, Martin Goddard, Pietro Lio, and Roger Foo. 2012. The landscape of DNA repeat elements in human heart failure. Genome Biol 13, 10 (2012), R90.

[12] Amy M Hauth and Deborah A Joseph. 2002. Beyond tandem repeats: complex pattern structures and distant regions of similarity. Bioinformatics 18, suppl 1 (2002), S31-S37.

[13] Fereydoun Hormozdiari, Can Alkan, Mario Ventura, Iman Hajirasouliha, Maika Malig, Faraz Hach, Deniz Yorukoglu, Phuong Dao, Marzieh Bakhshi, S Cenk Sahinalp, et al. 2010. Alu repeat discovery and characterization within human genomes. Genome research (2010).

[14] Ryan Koehler, Hadar Issac, Nicole Cloonan, and Sean M Grimmond. 2011. The uniqueome: a mappability resource for short-tag sequencing. Bioinformatics 27 , 2 (2011), 272-274.

[15] Sybille Kubis, Thomas Schmidt, and John Seymour PAT Heslop-Harrison. 1998. Repetitive DNA elements as a major component of plant genomes. Annals of Botany 82, suppl 1 (1998), 45-55.

[16] Stefan Kurtz. 2003. The Vmatch large scale sequence analysis software. Ref Type: Computer Program (2003), 4-12.

[17] Stefan Kurtz, Jomuna V Choudhuri, Enno Ohlebusch, Chris Schleiermacher, Jens Stoye, and Robert Giegerich. 2001. REPuter: the manifold applications of repeat analysis on a genomic scale. Nucleic acids research 29, 22 (2001), 4633-4642.

[18] Stefan Kurtz and Chris Schleiermacher. 1999. REPuter: fast computation of maximal repeats in complete genomes. Bioinformatics 15, 5 (1999), 426-427.

[19] Hayan Lee and Michael C Schatz. 2012. Genomic dark matter: the reliability of short read mapping illustrated by the genome mappability score. Bioinformatics 28, 16 (2012), 2097-2105.

[20] Heng Li. 2013. Aligning sequence reads, clone sequences and assembly contigs with BWA-MEM. arXiv preprint arXiv:1303.3997 (2013).

[21] Runsheng Li, Chia-Ling Hsieh, Amanda Young, Zhihong Zhang, Xiaoliang Ren, and Zhongying Zhao. 2015. Illumina synthetic long read sequencing allows recovery of missing sequences even in the "finished" C. elegans genome. Scientific reports 5 (2015), 10814.

[22] Wentian Li and Jan Freudenberg. 2014. Characterizing regions in the human genome unmappable by next-generation-sequencing at the read length of 1000 bases. Computational biology and chemistry 53 (2014), 108-117.

[23] Wentian Li and Jan Freudenberg. 2014. Mappability and read length. Frontiers in genetics 5 (2014)

[24] Wentian Li, Jan Freudenberg, and Pedro Miramontes. 2014. Diminishing return for increased Mappability with longer sequencing reads: implications of the k-mer distributions in the human genome. BMC bioinformatics 15, 1 (2014), 2.

[25] Guillaume Marçais and Carl Kingsford. 2011. A fast, lock-free approach for efficient parallel counting of occurrences of k-mers. Bioinformatics 27, 6 (2011), 764-770.

[26] Karen H Miga, Yulia Newton, Miten Jain, Nicolas Altemose, Huntington F Willard, and W James Kent. 2014. Centromere reference models for human chromosomes X and Y satellite arrays. Genome research 24, 4 (2014), 697-707.

[27] Suresh B Mudunuri and Hampapathalu A Nagarajaram. 2007. IMEx: imperfect microsatellite extractor. Bioinformatics 23, 10 (2007), 1181-1187.

[28] Alkes L Price, Neil C Jones, and Pavel A Pevzner. 2005. De novo identification of repeat families in large genomes. Bioinformatics 21, suppl_1 (2005), i351-i358.

[29] Astrid M Roy, Marion L Carroll, David H Kass, Son V Nguyen, Abdel-Halim Salem, Mark A Batzer, and Prescott L Deininger. 1999. Recently integrated human Alu repeats: finding needles in the haystack. Genetica 107, 1-3 (1999), 149-161.

[30] James A Shapiro and Richard von Sternberg. 2005. Why repetitive DNA is essential to genome function. Biological Reviews 80, 02 (2005), 227-250.

[31] EL Silva, RS Borba, and Patrícia Pasquali Parise-Maltempi. 2012. Chromosome mapping of repetitive sequences in Anostomidae species: implications for genomic and sex chromosome evolution. Molecular cytogenetics 5, 1 (2012), 1-8.

[32] Arian FA Smit, Robert Hubley, and Phil Green. 1996. RepeatMasker Open-3.0.

[33] Karen Usdin. 2008. The biological effects of simple tandem repeats: lessons from the repeat expansion diseases. Genome research 18, 7 (2008), 1011-1019. 